\title{
TABLE OF THE ZEROS AND WEIGHT FACTORS OF THE FIRST FIFTEEN LAGUERRE POLYNOMIALS
}

\author{
HERBERT E. SALZER AND RUTH ZUCKER
}

The chief use of the present table of zeros and weight factors of the Laguerre polynomials is in the performance of quadratures over the interval $[0, \infty]$, when the integrand behaves like the product of $e^{-x}$ by a polynomial. From the well known theory of orthogonal polynomials, the quadrature formula is exact for any polynomial $P(x)$ up to the $(2 n-1)$ th degree, provided one uses the $n$-point quadrature formula. Thus if $\alpha_{i}^{(n)}$ denotes the "weight factors" or "Christoffel numbers," corresponding to $L_{n}(x)$, the $n$th Laguerre polynomial, and $x_{i}^{(n)}$ denotes the zeros of $L_{n}(x)$, then

$$
\int_{0}^{\infty} e^{-x} P(x) d x=\sum_{i=1}^{n} \alpha_{i}^{(n)} P\left(x_{i}^{(n)}\right) .
$$

Besides problems involving direct quadratures, there are those arising in the numerical solution of linear integral equations, range $[0, \infty]$, where the unknown function occurs both inside and outside the integral sign. By considering the product of $e^{x}$ by the integrand as a polynomial, and making use of (1), the approximation problem reduces to the solution of a set of only $n$ linear equations. Hence only $n$ points are needed to give accuracy obtainable by approximating the product of $e^{x}$ by the integrand as a polynomial of the $(2 n-1)$ th degree. For a full description, including examples, see A. Reiz [3], ${ }^{1}$ especially pp. 1-12.

For many purposes, the report of the Admiralty Computing Service [2], which furnishes zeros to 8 decimals and weight factors to 8 significant figures as far as $L_{10}(x)$, will suffice. This present table is intended to cope with problems requiring higher degree and accuracy. Thus there are given here the zeros and weight factors of the first fifteen Laguerre polynomials, the zeros to 12 decimals and the weight factors to 12 significant figures. (The zeros and weight factors are available in manuscript form to two extra places for $n \leqq 10$, and to one extra place for $10<n \leqq 15$.) Also the example of A. Reiz is followed in that the quantities $\alpha_{i}^{(n)} e^{x_{i}(n)}$ are also tabulated to 12 significant figures, to facilitate the quadratures when the integrand does not contain $e^{-x}$ explicitly. Thus in

Received by the editors July 19, 1948.

${ }^{1}$ Numbers in brackets refer to the references cited at the end of the paper. 


$$
\int_{0}^{\infty} F(x) d x=\int_{0}^{\infty} e^{-x}\left[e^{x} F(x)\right] d x \sim \alpha_{i}^{(n)} e^{x_{i}^{(n)}} F\left(x_{i}^{(n)}\right),
$$

having $\alpha_{i}^{(n)} e^{x_{i}{ }^{(n)}}$ saves the labor of computing $e^{x_{i}{ }^{(n)}}$ and then multiplying $e^{x_{i}(n)}$ by either $\alpha_{i}^{(n)}$ or $F\left(x_{i}^{(n)}\right)$. In addition, the exact values of the coefficients of the first twenty Laguerre polynomials have been computed, because of their fundamental importance. ${ }^{2}$ They were calculated from the recursion formula (6) below, which was used to obtain $C_{m}^{(n+1)}$, the coefficient of $x^{m}$ in $L_{n+1}(x)$, by the relation

$$
C_{m}^{(n+1)}=(1+2 n) C_{m}^{(n)}-C_{m-1}^{(n)}-n^{2} C_{m}^{(n-1)},
$$

and as a check, it was verified that

$$
C_{m}^{(n)}=(-1)^{m}(n-m) !_{n} C_{n-m}^{2} .
$$

The Laguerre polynomial $L_{n}(x)$ is defined as

$$
L_{n}(x)=e^{x}\left[\frac{d^{n}}{d x^{n}}\left(e^{-x} x^{n}\right)\right]
$$

from which

$$
L_{n}(x)=(-1)^{n}\left(x^{n}-\frac{n^{2}}{1 !} x^{n-1}+\frac{n^{2}(n-1)^{2}}{2 !} x^{n-2}-\cdots\right)
$$

The polynomial $L_{n}(x)$ satisfied the orthogonality relations

$$
\int_{0}^{\infty} e^{-x} x^{i} L_{n}(x) d x=0, \quad i=0,1, \cdots, n-1 .
$$

Also, $L_{n}(x)$ satisfies the differential equation

$$
x L_{n}^{\prime \prime}(x)+(1-x) L_{n}^{\prime}(x)+n L_{n}(x)=0,
$$

and the recursion formula

$$
L_{n+1}(x)-(1+2 n-x) L_{n}(x)+n^{2} L_{n-1}(x)=0 .
$$

The weight factors $\alpha_{i}^{(n)}$ are given by

$$
\alpha_{i}^{(n)}=\frac{1}{L_{n}^{\prime}\left(x_{i}^{(n)}\right)} \int_{0}^{\infty} \frac{e^{-x} L_{n}(x)}{x-x_{i}^{(n)}} d x .
$$

The calculation of $\alpha_{i}^{(n)}$ is facilitated by the following relation (given in the Admiralty Report [2] and A. Reiz [3], the latter crediting it tory.

2 These coefficients are available in manuscript form at the Computation Labora- 
to J. Deruyts [6]):

$$
\int_{0}^{\infty} \frac{e^{-x} L_{n}(x)}{x-x_{i}^{(n)}} d x=\frac{(n !)^{2}}{x_{i}^{(n)} L_{n}^{\prime}\left(x_{i}^{(n)}\right)}
$$

from which there follows the very convenient formula for $\alpha_{i}^{(n)}$, namely,

$$
\alpha_{i}^{(n)}=\frac{1}{x_{i}^{(n)}}\left[\frac{n !}{L_{n}^{\prime}\left(x_{i}^{(n)}\right)}\right]^{2} .
$$

The zeros of $L_{n}(x)$ were calculated by Newton's method, from a first approximation. In most cases, for a given $n$, a first approximation to $x_{i}^{(n)}$ was obtained by either extrapolation as a function of $n$ for the same value of $i$, or when $i$ was considerably greater than 1 , by extrapolation for fixed $n$, based upon the previous values of $i$ (or a combination of both these processes). Then the first approximation was refined still further by 1 ), noting by how much previously obtained roots differed from their values obtained by this method of extrapolation, and 2), using another operation of extrapolation upon those deviations to find the deviation of the first approximation. All roots were checked by substitution into the polynomials $L_{n}(x)$, and noting how closely they satisfied $L_{n}\left(x_{i}^{(n)}\right)=0$. In addition the roots were checked by the relations

$$
\sum_{i=1}^{n} x_{i}^{(n)}=n^{2}
$$

and

$$
\prod_{i=1}^{n} x_{i}^{(n)}=n !
$$

The first step in finding the weight factors (or Christoffel numbers) was to obtain $L_{n}^{\prime}\left(x_{i}^{(n)}\right)$. Because the calculations of $L_{n}^{\prime}\left(x_{i}^{(n)}\right)$ were lengthy, as a check upon $L_{n}^{\prime}\left(x_{i}^{(n)}\right)$, the second derivatives $L_{n}^{\prime \prime}\left(x_{i}^{(n)}\right)$ were also computed, and were employed in the checking equation

$$
x_{i}^{(n)} L_{n}^{\prime \prime}\left(x_{i}^{(n)}\right)=\left(x_{i}^{(n)}-1\right) L_{n}^{\prime}\left(x_{i}^{(n)}\right) .
$$

Then the weight factors $\alpha_{i}^{(n)}$ were calculated from (9) and checked by the relation $(n !)^{2}=\alpha_{i}^{(n)} x_{i}^{(n)}\left\{L_{n}^{\prime}\left(x_{i}^{(n)}\right)\right\}^{2}$, and also by substitution into (1), with $P(x)=x^{m}$, where $m$ ! must be obtained. For each $L_{n}(x), m$ was taken successively as every number in the set $0,1,2,4,8,16$, and 24 which did not exceed $2 n-1$. This wide range of $m$ was necessary in order to be sure of including all the desired significant figures 
of each $\alpha_{i}^{(n)}$ in the checking.

The quantities $e^{x_{i}{ }^{(n)}}$ were obtained by interpolation in the Mathematical Tables Project's Tables of the exponential function $e^{x}$ and checked by interpolation in the Project's Table of natural logarithms, vols. III and IV. Finally, to guarantee the quantities $\alpha_{i}^{(n)} e^{x_{i}{ }^{(n)}}$, they were checked both by division by $e_{i}^{x_{i}^{(n)}}$, and by duplicate calculation.

Zeros of Laguerre
Polynomials

$$
n=1
$$

1.000000000000

$n=2$

$\begin{array}{llll}.58578 & 64376 \quad 27\end{array}$

3.414213562373

$n=3$

.415774556783

$2.2942803602 \quad 79$

6.289945082937

$$
n=4
$$

$\begin{array}{rrr}.32254 & 76896 & 19 \\ 1.74576 & 11011 & 58 \\ 4.53662 & 02969 & 21 \\ 9.39507 & 09123 & 01\end{array}$

$n=5$

$.2635603197 \quad 18$

$\begin{array}{llll}1.41340 & 30591 \quad 07\end{array}$

$3.59642 \quad 5771041$

7.085810005859

$12.64080 \quad 0844276$

$$
n=6
$$

$.22284 \quad 6604179$

1.188932101673

2.992736326059

5.775143569105
Weight Factors ${ }^{3}$

$$
n=1
$$

1.000000000000

$$
n=2
$$

.853553390593 .146446609407

$$
n=3
$$

.711093009929

$$
.278517733569
$$

.(1) 103892565016

$n=4$

.603154104342

.357418692438

.(1) 388879085150

.(3) 539294705561

$$
n=5
$$

.521755610583 .398666811083

.(1) $7594244968 \quad 17$

.(2) 361175867992

.(4) 233699723858

$\begin{array}{ccc}n=6 & & \\ .45896 & 46739 & 50 \\ .41700 & 08307 & 72 \\ .11337 & 33820 & 74 \\ . \text { (1) } 10399 & 19745 & 31\end{array}$

Weight Factors

$\times$ Exponential of Zeros

$$
n=1
$$

2.71828182846

$$
n=2
$$

$\begin{array}{llll}1.53332 & 603312\end{array}$

4.45095733505

$$
n=3
$$

1.07769285927

2.76214296190

5.60109462543

$$
n=4
$$

.832739123838

2.04810243845

3.63114630582

6.48714508441

$$
n=5
$$

.679094042208

1.63848787360

2.76944324237

4.31565690092

$7.2191863543 \quad 5$

$$
n=6
$$

.573535507423

1.36925 259071

2.26068459338

3.35052458236

3 The number in the parentheses stands for the number of zeros between the decimal point and the first significant figure. 
Zeros of Laguerre Polynomials

$$
n=6
$$

9.837467418383 15.982873980602

$$
n=7
$$

.193043676560

$1.0266648953 \quad 39$

$2.56787 \quad 67449 \quad 51$

4.900353084526

8.182153444563 12.734180291798 $19.39572 \quad 7862263$

$$
n=8
$$

.170279632305

.903701776799

2.251086629866 $4.2667001702 \quad 88$ 7.045905402393 10.758516010181

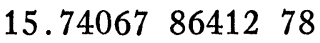
$22.86313 \quad 17368 \quad 89$

$$
n=9
$$

$.15232 \quad 22277 \quad 32$ .807220022742 $2.0051351556 \quad 19$ $\begin{array}{llll}3.78347 & 39733 & 31\end{array}$ 6.204956777877 9.372985251688 $13.46623 \quad 6911092$ 18.833597788992 26.374071890927

$$
n=10
$$

$.1377934705 \quad 40$ .729454549503
Weight Factors ${ }^{3}$

$$
n=6
$$

(3) 261017202815

.(6) 898547906430

$$
n=7
$$

.409318951701 .421831277862 .147126348658

.(1) 206335144687

(2) 107401014328

.(4) 158654643486

.(7) 317031547900

$$
n=8
$$

.369188589342 .418786780814 .175794986637

. (1) 333434922612

. (2) 279453623523

. (4) 907650877336

.(6) 848574671627

.(8) 104800117487

$$
n=9
$$

.336126421798 .411213980424 .199287525371

.(1) 474605627657

.(2) 559962661079

. (3) 305249767093

.(5) 659212302608

.(7) 411076933035 .(10) 329087403035

$$
n=10
$$

.308441115765 .401119929155
Weight Factors $\times$ Exponential of Zeros

$$
n=6
$$

4.88682680021 7.84901594560

$$
n=7
$$

.496477597540 1.17764306086 1.91824978166 $2.77184 \quad 863623$ 3.84124912249 5.38067820792 8.40543248683

$$
n=8
$$

.437723410493 1.03386934767 1.66970976566 2.37692470176 3.20854091335 4.26857551083 5.81808336867 8.90622621529

$$
n=9
$$

.391431124316 .921805028529 1.48012790994 $2.0867708075 \quad 5$ $2.7729213897 \quad 1$ 3.59162606809 4.64876600214 6.21227541975 $9.3632182377 \quad 1$

$$
n=10
$$

.354009738607 .831902301044 
1949] ZEROS AND WEIGHT FACTORS OF LAGUERRE POLYNOMIALS 1009

Zeros of Laguerre

Polynomials

$$
n=10
$$

$1.8083429017 \quad 40$

3.401433697855

5.552496140064

$8.3301527467 \quad 64$

11.843785837900

16.279257831378

21.996585811981

29.920697012274

$$
n=11
$$

.125796442188

.665418255839

1.647150545872

3.091138143035

5.029284401580

$\begin{array}{llll}7.50988 & 78638 \quad 07\end{array}$ $10.60595 \quad 09995 \quad 47$ 14.431613758064 19.178857403215 $25.21770 \quad 93396 \quad 78$ 33.497192847176

$$
n=12
$$

.115722117358

.611757484515

$\begin{array}{lll}1.51261 & 02697 \quad 76\end{array}$

$2.8337513377 \quad 44$

4.599227639418

$6.8445254531 \quad 15$

$9.62131 \quad 68424 \quad 57$

13.006054993306

17.116855187462

$22.1510903793 \quad 97$

28.487967250984 37.099121044467
Weight Factors ${ }^{3}$

$$
n=10
$$

.218068287612

.(1) 620874560987

.(2) 950151697518

.(3) 753008388588

.(4) 282592334960

.(6) 424931398496

.(8) 183956482398

.(12) 991182721961

$$
n=11
$$

.284933212894 .389720889528 .232781831849

.(1) 765644535462

.(1) 143932827674

.(2) 151888084648

. (4) 851312243547

.(5) 229240387957

.(7) 248635370277

(10) 771262693369

.(13) 288377586832

$$
n=12
$$

$.26473 \quad 1371055$ .377759275873 .244082011320

.(1) 904492222117

.(1) 201023811546

.(2) 266397354187

.(3) 203231592663

.(5) 836505585682

.(6) 166849387654

.(8) 134239103052

.(11) 306160163504 .(15) 814807746743
Weight Factors $\times$ Exponential of Zeros

$$
n=10
$$

\subsection{5 \\ 1.86306390311 \\ 2.45025555808 \\ 3.12276415514 \\ 3.93415269556 \\ 4.99241487219 \\ 6.57220248513 \\ $9.7846958403 \quad 7$}

$$
n=11
$$

.323128880435

$.7581255998 \quad 10$

1.20864142290

1.68457916714

2.19963347862

$2.77348 \quad 97457 \quad 5$

3.43712141652

4.24484029080

5.30682601948

$6.9042154788 \quad 3$

10.1767127469

$$
n=12
$$

.297209636044 .696462980431

1.10778139462

1.53846423904

1.99832760627

2.50074576910

3.06532151828

3.72328911078

4.52981402998

$5.59725 \quad 84618 \quad 4$

7.21299546093

10.5438374619 
Zeros of Laguerre Polynomials

$$
n=13
$$

.107142388472 .566131899040 1.398564336451 2.616597108406 4.238845929017 6.292256271140 8.815001941187 11.861403588811 $15.51076 \quad 2037704$ $19.88463 \quad 5663880$ 25.185263864678 31.800386301947 40.723008669266

$$
n=14
$$

$.09974 \quad 7507033$ .526857648852

1.300629121251 $2.4308010787 \quad 31$ $3.93210 \quad 2822293$ $5.82553 \quad 6218302$ 8.140240141565 10.916499507366 14.210805011161 $18.1048922202 \quad 18$ $22.72338 \quad 1628269$ $28.27298 \quad 17232 \quad 48$ 35.149443660592 44.366081711117

$$
n=15
$$

$\begin{array}{lll}.09330 \quad 78120 & 17\end{array}$ .492691740302 1.215595412071 2.26994 9526204 $3.66762 \quad 2721751$ $5.42533 \quad 66274 \quad 14$
Weight Factors ${ }^{3}$

$$
n=13
$$

$.24718 \quad 8708430$ .365688822901 .252562420058 .103470758024

.(1) 264327544156

.(2) 422039604027

.(3) 411881770473

.(4) $2351547398 \quad 15$

.(6) 731731162025

.(7) 110884162570 .(10) 677082669221 .(12) 115997995991 .(16) 224509320389

$$
n=14
$$

.231815577145 .353784691598 .258734610245 $.11548 \quad 2893557$

.(1) 331920921593

.(2) 619286943701

.(3) $7398903778 \quad 67$

.(4) 549071946684

.(5) 240958576409

.(7) 580154398168

.(9) 681931469249

.(11) 322120775189 .(14) 422135244052 .(18) 605237502229

$$
n=15
$$

.218234885940 .342210177923 $.26302 \quad 7577942$ .126425818106 .(1) 402068649210 .(2) 856387780361
Weight Factors $\times$ Exponential of Zeros

$$
n=13
$$

.275143955471 .644139076543

$1.02272 \quad 177858$

1.41641760566

1.83252461786

2.28058038229

2.77382636586

3.33192995643

3.98648297492

4.79354782193

5.86763133711

7.50209880492

10.8896100139

$$
n=14
$$

.256131154737 $.5991704774 \quad 95$ $.94997 \quad 1502475$

1.31280781147 1.69326599075 $2.09840 \quad 908427$ 2.53763265494 3.02415991770 $3.5778043557 \quad 2$ 4.23056503491 5.03941336846 6.12094792833 7.77429536701 11.2168342167

$$
n=15
$$

.239578170311 $.56010 \quad 0842793$ .887008262919

1.22366440215

1.57444872163 1.94475197653 


\section{Zeros of Laguerre Polynomials}

$$
n=15
$$

Weight Factors ${ }^{3}$

$$
n=15
$$

.(2) 121243614721

.(3) 111674392344

.(5) 645992676202

.(6) 222631690710

.(8) 422743038498

.(10) 392189726704

.(12) 145651526407

.(15) 148302705111 .(19) 160059490621
Weight Factors $\times$ Exponential of Zeros

$$
n=15
$$

2.34150205664

2.77404192683

3.25564334640

3.80631171423

$4.4584777538 \quad 4$

$5.2700177844 \quad 3$

6.35956346973

8.03178763212

11.5277721009

\section{BIBLIOGRAPHY}

Note: A detailed bibliography on Laguerre polynomials is really unnecessary, since a very exhaustive one up to the year 1940 is given in J. Shohat [1]. However, the few references given below afford a representative sample and cover many essential aspects of Laguerre polynomials.

1. J. A. Shohat, $A$ bibliography on orthogonal polynomials, Bulletin of the National Research Council, no. 103, 1940. Lists over 2000 articles, books, and theses, by 643 authors, on the subject of orthogonal polynomials. The topical index has a special section on Laguerre polynomials, where more than 250 references are given.

2. Department of Scientific Research and Experiment, Admiralty Computing Service, Zeros of Laguerre polynomials and the corresponding Christoffel numbers, June, 1945.

3. A. Reiz, On the numerical solution of certain types of integral equations, Arkiv för Matematik, Astronomi och Fysik, vol. 29A, no. 29.

4. G. Szegö, Orthogonal polynomials, Amer. Math. Soc. Colloquium Publications, vol. 23, 1939. Includes a large bibliography.

5. D. Jackson, Fourier series and orthogonal polynomials, Carus Mathematical Monographs, no. 6, Mathematical Association of America, 1941. Chapters VII (general) and $X$ (on Laguerre polynomials).

6. J. Deruyts, Sur le calcul approché de certaines intégrales définies, Bulletins de l'Académie Royale de Belgique (3) vol. 11 (1886) pp. 307-311.

7. W. Hahn, Bericht über die Nullstellen der Laguerreschen und der Hermiteschen Polynome, Jber. Deutschen Math. Verein. vol. 44 (1934) pp. 215-236. An elaborate bibliography is included in this paper.

8. E. Moecklin, Asymptotische Entwicklungen der Laguerreschen Polynome, Comment. Math. Helv. vol. 7 (1934) pp. 24-46.

9. F. Tricomi, Generalizzazione di una formula asintotica sui polinomi di Laguerre e sue applicazioni, Atti Accad. Sci. Torino vol. 76 (1941) pp. 288-316.

10. E. C. Kemble, The fundamental principles of quantum mechanics, McGrawHill, 1937, pp. 160, 585-587. 
11. J. Cossar and A. Erdélyi, Dictionary of Laplace transforms, Part 2B (issued by Dept. of Scientific Research and Experiment, Admiralty Computing Service), pp. 84-85.

12. H. Margenau and G. M. Murphy, The mathematics of physics and chemistry, New York, Van Nostrand, 1943, pp. 77-78, 122-128, 350.

13. R. Courant and D. Hilbert, Methoden der mathematischen Physik, vol. I, Berlin, Springer, 1931, pp. 79-80, 284-285, 294-296, 324-326, 440-441. (Reprinted by Interscience Publishers, 1943.)

14. N. Kosliakov, The calculation by a formula of mechanical quadrature of definite integrals with infinite limits (Russian), Bull. Acad. Sci. URSS. (7) (1933) pp. 801808.

Of the above references, [2], [3], and [14] include zeros and Christoffel numbers. Those in [2] agree exactly with the table above as far as comparison can be made. The zeros and Christoffel numbers in [3], both given to $7 D$, up to $n=5$, also agree with this table as far as comparison can be made, save for a few values that differ by no more than 2 units in the last place. The zeros and Christoffel numbers in [14], given only for $n=5$, to $7 D$, are all incorrect (except for $\left.A_{4}\right)$ up to as much as 63 units in the 7 th decimal place. For details on [14], see MTAC III, no 21, Jan. 1948, item 121 on p. 41. In a footnote to [9], there are given 5-decimal values of the zeros of the first few Laguerre polynomials, correct to within several units of the last place. But in the text on p. $308, x_{10}^{(10)}$ is erroneously given as 29.9315 instead of 29.9207. In [3], there occurs $\alpha_{i}^{(n)} e^{x_{i}{ }^{(n)}}$, up to $n=5$, to $7 D$.

Computation Laboratory, National Bureau of Standards 